\title{
Mixed Traffic Flow Model with Cellular Automata
}

\author{
Shuo Yang
}

State Key Laboratory of Rail Traffic Control and Safety, Beijing Jiaotong University, Beijing 100044, China

15120904@bjtu.edu.cn

Keywords: Urban road; Traffic flow; Cellular automata; Lateral interference

\begin{abstract}
Urban transportation with multiple traffic modes is quite a complex system, where pedestrian-vehicle mixed flows play important roles. This paper is to analyze the effect of pedestrians' crossing street on vehicle flows based on the investigation of their traffic characteristics. Rules of lane changing, acceleration, deceleration, randomization and update are modified here to simulate real traffic flows better. Then an urban two-lane cellular automata model of traffic flows is established under the above rules. MATLAB is used for numerical simulation of different traffic conditions. Meanwhile, space-time diagram and relational graphs of traffic flow parameters are generated and then comparatively analyzed. The analyzation results illustrate that the proposed model is in good consistence with real situation and can provide some practical reference for urban traffic management.
\end{abstract}

\section{Introduction}

Cellular Automata model (referred to as CA model) is a mathematical model with discrete time and space units, which can be well applied to simulation of microscopic traffic flow phenomena. Since the classic one-dimensional CA traffic flow model - NS model [1] was proposed by Nagel and Schreckenberg in 1992, many scholars had established some CA models considering different road and traffic conditions by improving individual vehicle rules. Recent research [2-5] in this field focuses primarily on the impact of traffic bottleneck sections as well as mixed traffic flow. Existing research [6-9] involves in CA models in terms of mixed traffic flow, driver psychology, longitudinal interference or other factors.

Pedestrian-vehicle mixed traffic flows account for a big proportion in urban traffic flows in China, where crossing street of considerable pedestrians on urban segments becomes a typical feature nowadays and has a direct impact on the continuity of traffic flow. Since characteristics of pedestrians crossing on uncontrolled low-grade roads or branch roads without separating barriers in cities have received little attention for now, only a few scholars have respectively conducted several studies [10-12] concerning section crosswalks.

In view of self-organizing behaviours of pedestrians and vehicles at crosswalk, considering psychological characteristics of pedestrians and motor vehicle drivers, in this paper we established a CA model mainly focusing on characteristics of vehicles under lateral interference of pedestrians around non-signal-controlled crosswalk. Based on the simulation data, relationships between vehicle speed, traffic density, traffic flux, arrival rate of pedestrians and other relative parameters are respectively researched.

This paper is organized as follows. In section 2, we will describe our model in detail. Then numerical simulation is presented to demonstrate the proposed model in section 3. Finally, we summarise and conclude the paper in section 4.

\section{Formulation}

We established a CA model which can be illustrated from views of vehicle movement, pedestrian movement and vehicle-pedestrian conflict.

Vehicle Movement. Two-lane traffic flow CA models without lateral pedestrian interference have been advanced, which can be used here after modification. Lane changing rules are supposed 
to involve in road conditions, traffic conditions as well as drivers' psychological property and other related factors. This model applies a two-step method within each discrete time unit to renew vehicles' position and speed condition.

First Step: Lane changing

At crosswalks vehicle's behaviours are quite complex when approaching the conflict areas. If pedestrians invade a small space of the lanes, vehicles probably transfer tracks to keep a safe distance from pedestrians, causing some tortuous journeys. If pedestrians occupy most of current lane's space, vehicles are likely to slow to a halt. Besides, drivers usually do not change lanes on crosswalks.

Second Step: Speed and location updating

(1) Acceleration: Drivers tend to drive at as high speed as possible when traffic conditions permit.

$v_{n}(t+1)=\min \left(v_{n}(t)+1, v_{\max }\right)$

(2) Deceleration: Drivers have to curb speed to avoid collisions with the front vehicle.

$v_{n}(t+1)=\min \left(v_{n}(t), d_{n}-1\right)$

(3) Randomization: Vehicles slow down because of varieties of uncertain factors such as terrible road conditions or different drivers' mentality.

If $\operatorname{rand}()<p_{0}$, then $v_{n}(t+1)=\max \left(v_{n}(t+1)-1,0\right)$

(4) Location updating: The car advances $v_{n}(t+1)$ cells within each time unit.

$$
x_{n}(t+1)=x_{n}(t)+v_{n}(t+1)
$$

Pedestrian Movement. We use one cell to represent a pedestrian and the surrounding eight cells have close relationship with it. Pedestrian mobile matrix is also organized as the grid. We referred to papers [11] and [12] combining six parameters into the mobile matrix that determines which cell around the pedestrian it would move into. The value of each number in the matrix represents the attractiveness of corresponding cell to the pedestrian. The six parameters are as follows:

(1) Occupation-parameter: This parameter is set to restrict pedestrians from moving into a cell which is already occupied. An empty cell's value of this parameter is set 0 , while an occupied cell's is -1 .

(2) Safety-parameter: Pedestrians decide whether to step forward mainly according to vehicles' speed and distance between vehicles and themselves, symbolled with a certain probability in the simulation.

(3) Benefit-parameter: Generally speaking, pedestrians wish to move forward as quickly as possible while crossing street. Accordingly, to move towards the front cell will gain the benefit of 1 unit, while $0.7(1 / \sqrt{ } 2 \approx 0.7)$ can be achieved if stepping into the front-left cell or front-right cell.

(4) Attraction-parameter: Pedestrians prefer to cross streets within a flock of the same direction, which is a typical form of herd behaviors. So the attraction-parameter is proposed to describe the attraction within a certain affected area.

(5) Horizontal-parameter: It can be frequently observed that people are used to staying in a row when crossing streets out of safety concerns. We use the following formula to determine the horizontal-parameter matrix.

(6) Forward-parameter: People would like to move towards open areas when crossing in case that it would be too crowded to choose their next easy foothold.

The above six parameters jointly contribute to the final decision of which cell pedestrians will step into at the next time unit.

Vehicle-Pedestrian Conflict. When threshold of acceptable crossing gaps for pedestrians is exceeded by distance from the closest coming vehicle to walkers on roads, pedestrians will occupy lanes and take the chance to cross. Otherwise, pedestrians will go ahead by a certain probability. 
Moreover, the larger the number of pedestrians waiting on the roadside is, the greater the probability of vehicles' decelerating and giving way to pedestrians will be.

\section{Simulation Analyses}

The Basic Setup of Numerical Simulation. A single stride length of an individual crossing a road is surveyed to be about 0.6 meters, so the cellular grid length is set to be 0.6 meters in the models. Each time unit represents 0.5 seconds. Open boundary condition is used for numerical simulation: namely vehicles and pedestrians reach the boundaries of roads at certain arrival rates. The composition of our model can be depicted in Fig. 1.

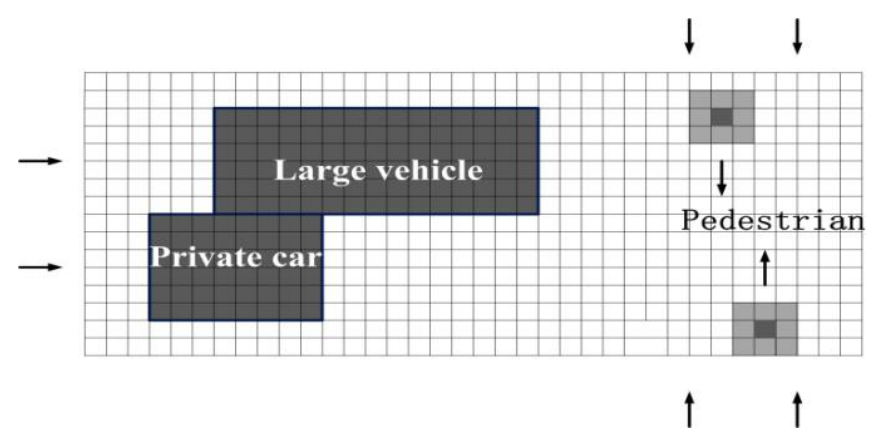

Figure 1. Sketch of elements in the model

Density of lane $j$ at time $t$ is defined as $\rho_{j}(t)=N_{j}(t) / L$, while average speed $V=\frac{1}{T} \sum_{t=t_{0}}^{t_{0}+T-1} \bar{V}_{t}$, average flow $Q=\rho \times V$. Where $N$ is the total number of vehicles, $L$ is the total cellular number on single lane, $N_{j}(t)$ is the vehicle number on lane $j$ at time $t, \bar{V}_{t}$ is the average speed of vehicle on a lane at time $t, T$ is total time of each simulation.

Numerical Simulation Results Analysis. Fig. 2-5 are vehicles' space-time (S-T) diagrams for single lane with different arrival rates of vehicles $\left(\lambda_{\mathrm{v}}\right.$, unit: veh/(s·lane) $)$ and pedestrians $\left(\lambda_{\mathrm{p}}\right.$, unit: $\mathrm{ped} /(\mathrm{h} \cdot \mathrm{m}))$. In these figures, horizontal axes represent the movement of vehicles in space, and vertical axes show the movement of them in temporal dimension. Moreover, the colorful short dashes signify vehicles' position while colorful patches stand for delay of these vehicles.

Fig. 2 manifests S-T characteristics of sections without pedestrian disturbing. Observation can find in lower density, for example when $\rho=12.2\left(\mathrm{veh} /(\mathrm{km} \cdot \mathrm{lane}), \lambda_{\mathrm{v}}=0.16\right)$, that vehicles travel in free flow state and are quite evenly distributed. In higher density of $\rho=51.6$, vehicles compose a rather crowded traffic, travelling in a synchronize flow state. Queues are formed and road space is unevenly utilized. The above phenomena are consistent with existing research conclusions. What's more, dense but not crowded conditions appear at specific time due to randomness.

Fig. 3-5 depict traffic conditions on non-signalized sections with crosswalk. In lower density such as $\rho=14.3$, some small queues and blockage is quickly dissipated, which can be clearly found out in Fig. 3. In higher density but with less disturb of pedestrians, intensive vehicles become a bit vulnerable to occasional queues caused by pedestrians at crosswalk as is shown in Fig. 4. In Fig. 5 with heavier interference in high density vehicles are driving in a very crowded state, where longer queues are appearing almost continuously. Thus road resource is even less efficiently assigned after the crosswalk. 


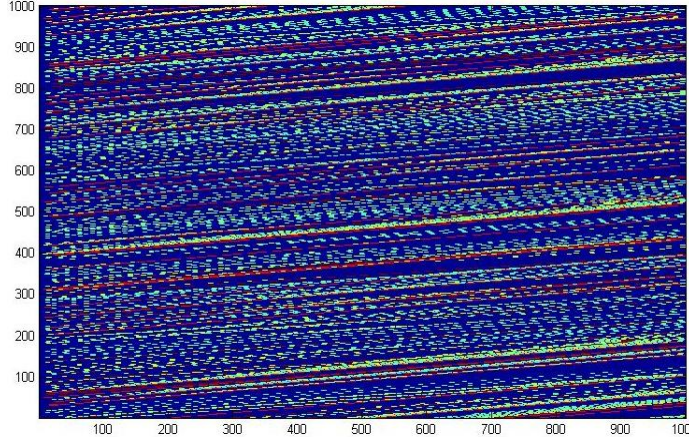

Figure 2. S-T diagram of sections without interference in higher density $(\lambda p=0, \lambda v=1.0, \rho=51.6)$

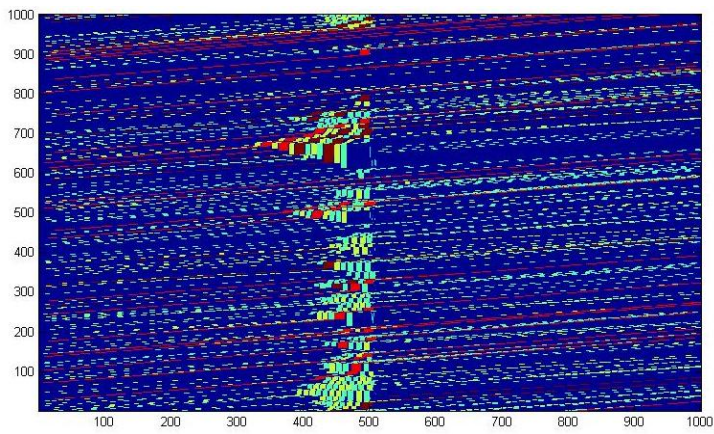

Figure 4. S-T diagram of sections with slight interference in higher density $(\lambda p=108, \lambda v=0.4, \rho=30.1)$

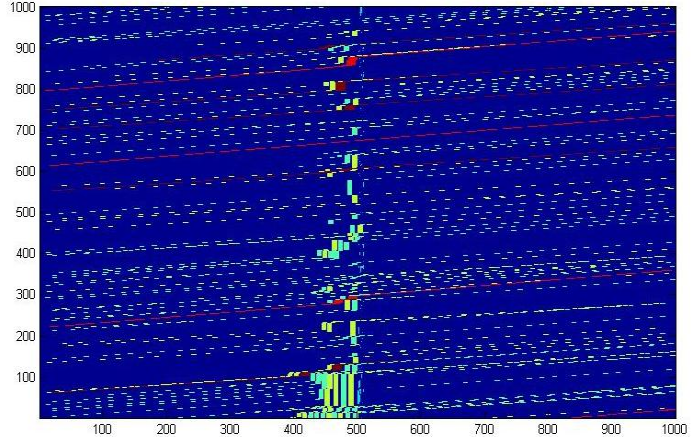

Figure 3. S-T diagram of sections with heavy interference in moderate density $(\lambda \mathrm{p}=168, \lambda \mathrm{v}=0.16, \rho=14.3)$

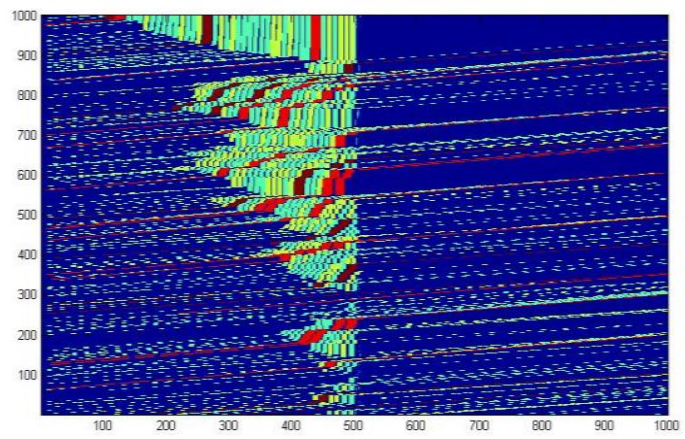

Figure 5. S-T diagram of sections with heavy interference in higher density $(\lambda \mathrm{p}=168, \lambda \mathrm{v}=0.4, \rho=45.2)$

Fig. 6-7 reflect how traffic density or average velocity changes with the arrival rates of pedestrians. We can see that moderate lateral interference has less impact on both traffic density and average velocity, while as quantities of pedestrians increase, changes in traffic conditions get increasingly bigger and slower and more crowded flows come into being. That is because, when a small number of pedestrians cross street, acceptable crossing gaps are utilized so that they only have a slight influence on vehicles' states of motion.

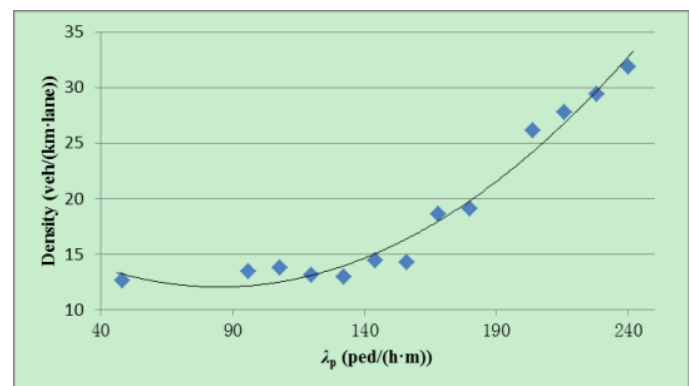

Figure 6. Relationships between traffic density and arrival rates of pedestrians

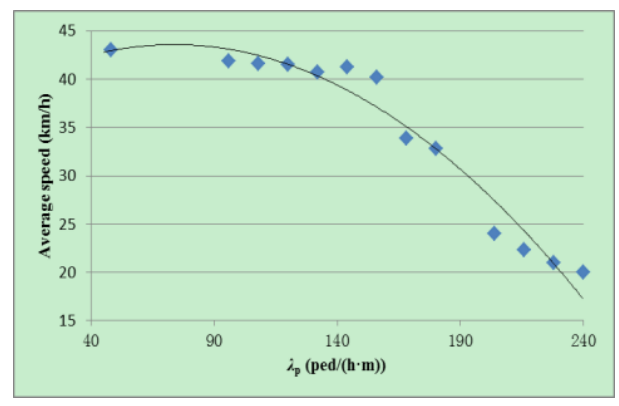

Figure 7. Relationships between traffic average speed and arrival rates of pedestrians

\section{Conclusions}

This paper has come to the following three conclusions: 
(1) We established a traffic flow cellular automaton model of urban two-lane road concerning pedestrians crossing streets at crosswalks without signal control. Space-time diagrams are used to reproduce traffic congestion, queuing and dissipation phenomena under different road conditions. We deduced some specific traits on different conditions from these diagrams.

(2) Numerical simulation results indicate that when arrival rate of pedestrians is moderate (about less than $6 \mathrm{ped} /(\mathrm{h} \cdot \mathrm{m})$ ) pedestrians' crossing street has slight impact on traffic flow. As the rate increases, changes in traffic conditions get increasingly bigger as slower and more crowded flows come into being on the involved sections.

(3) We can also deduce that when vehicle density is lower than around $25 \mathrm{veh} /(\mathrm{km} \cdot \mathrm{lane})$, pedestrians have modest impact on traffic flow, whereas when vehicle density is higher than about $60 \mathrm{veh} /(\mathrm{km} \cdot \mathrm{lane})$, traffic speed and volume will decrease significantly especially on sections with non-signal-controlled crosswalk.

\section{References}

[1] K. Nagel, M. Schreckenberg: A cellular automaton model for freeway traffic, Journal of Physics I France, Vol. 2 (1992) No.12, p.2221-2229.

[2] M. Zamith, R.C.P. Leal-Toledo, M. Kischinhevsky, E. Clua, D. Brandão, A. Montenegro, E.B. Lima: A probabilistic cellular automata model for highway traffic simulation, Procedia Computer Science, Vol. 1(2010) No.1, p.337-345.

[3] P. Korcek, L. Sekanina, O. Fucik: A scalable cellular automata based microscopic traffic simulation, 2011 IEEE Intelligent Vehicles Symposium, Vol. 30(2011) No.1, p.13-18.

[4] S. Das: Cellular automata based traffic model that allows the cars to move with a small velocity during congestion, Chaos Solitons \& Fractals, Vol. 44(2011) No.44, p.185-190.

[5] C. Appert-Rolland and J. D. Boisberranger: Macroscopic relaxation after on-ramps in real data and in cellular automata simulations, Transportation Research Part C: Emerging Technologies, Vol.34 (2013) No.9, p.162-175.

[6] X.G. Li: Microscopic modeling and properties analyzing of transportation system based on cellular automata model (Ph.D., Beijing Jiaotong University, China 2009). (in Chinese)

[7] X.D. Hua, W. Wang, H. Wang: A two-lane cellular automaton traffic flow model with the influence of driving psychology. Acta Physica Sinica, Vol. 60(2011) No.8, p.298-300. (in Chinese)

[8] Y.S. Qian, H.L. Wang: New cellular automaton city traffic model considering public transport Vehicles affect with mixed traffic flow. Journal of System Simulation, Vol. 19(2007) No.14, p.3358-3360. (in Chinese)

[9] M.E. Lárraga, L. Alvarez-Icaza: Cellular automaton model for traffic flow based on safe driving policies and human reactions. Physica A, Vol. 389 (2010) No.23, p.5425-5438.

[10]J.H. Wang, S.E. Fang: Pedestrian-vehicle conflict observation and characteristics of road section. Journal of Tongji University, Vol.36 (2008) No.4, p.503-507. (in Chinese)

[11]H. Yue: Study on Simulation Model of Pedestrian Flow Based on Cellular Automata (Ph.D., Beijing Jiaotong University, China 2008). (in Chinese)

[12]S.M. Feng, N. Ding, T. Chen, H. Zhang: Simulation of pedestrian flow based on cellular automata: A case of pedestrian crossing street at section in China. Physica A Statistical Mechanics \& Its Applications, Vol.392 (2013) No.13, p.2847-2859. 